\title{
On Partial Poincaré Series
}

\author{
J.W. Cogdell and I.I. Piatetski-Shapiro
}

This paper is dedicated to our colleague and friend Steve Gelbart.

\begin{abstract}
The theory of Poincaré series has played a central role in the theory of automorphic forms and their applications. For the analysis of Fourier coefficients for example one deals with a Poincaré series formed with functions that have a broad spectral "footprint". For the converse theorem one would like to make a similar construction but beginning with a function having a small spectral footprint. For such functions one cannot form a full Poincaré series, but only what we call a partial Poincaré series. In this note we recall the partial Poincaré series on $\mathrm{GL}_{n}(\mathbb{A})$ that play a role in the converse theorem and show that they are rapidly decreasing automorphic functions on the embedded $\mathrm{GL}_{n-1}(\mathbb{A})$. It is then the purpose of the converse theorem to determine when these partial Poincaré series are actually cuspidal automorphic forms on $\mathrm{GL}_{n}(\mathbb{A})$.
\end{abstract}

\section{Introduction}

The theory of Poincaré series has played a central role in the theory of automorphic forms and their applications. For the analysis of Fourier coefficients, one deals with a Poincaré series of the following type. Let us work with the group $G=\mathrm{GL}_{n}$ over a number field $k$. Let $\mathbb{A}$ denote the adele ring of $k$ and let $\psi$ be a non-trivial additive character of $\mathbb{A}$ which is trivial on $k$. Let $N=\mathrm{N}_{n}$ denote the standard maximal unipotent subgroup of $\mathrm{GL}_{n}$ realized as upper triangular unipotent matrices. Extend $\psi$ to a non-degenerate character of $\mathrm{N}_{n}(\mathbb{A})$ through its abelianization

$$
\mathrm{N}_{n}(\mathbb{A}) \rightarrow \mathrm{N}_{n}(\mathbb{A}) /\left[\mathrm{N}_{n}(\mathbb{A}), \mathrm{N}_{n}(\mathbb{A})\right] \simeq \mathbb{A}^{n-1} \stackrel{\psi}{\longrightarrow} \mathbb{C}^{\times} .
$$

In coordinates, if $u=\left(u_{i, j}\right) \in \mathrm{N}_{n}$ then $\psi(u)=\psi\left(u_{1,2}+\cdots+u_{n-1, n}\right)$. Consider the space $S(N \backslash G ; \psi)$ consisting of all smooth functions on $G(\mathbb{A})$ that satisfy $f(u g)=$ $\psi(u) f(g)$. Note that the functions in this spaces are left invariant under the rational points of $N$, i.e., under $N(k)$. Given a function $f \in S(N \backslash G ; \psi)$ one forms an associated Poincaré series $P_{f}(g)$ by

$$
P_{f}(g)=\sum_{\gamma \in N(k) \backslash G(k)} f(\gamma g)
$$

The first author was supported in part by various NSA and NSF grants.

The second author was supported in part by various NSF grants. 
when this converges. In practice, for example in $[\mathbf{1}],[\mathbf{2}],[\mathbf{5}]$, and $[\mathbf{8}]$, the functions $f(g)$ take a specific form dictated by the application in mind and the convergence argument essentially follows that for Eisenstein series. In these applications it is important that the Poincaré series, and hence the input functions $f(g)$, have a broad spectral "footprint".

For the converse theorem $[\mathbf{3}, \mathbf{4}]$ one would like to make a similar construction but beginning with a function having a small spectral footprint. One begins with an irreducible admissible representation $\Pi \simeq \otimes^{\prime} \Pi_{v}$ of $\mathrm{GL}_{n}(\mathbb{A})$ satisfying certain mild coherence conditions: its central character should be automorphic and its formally defined $L$-function $L(s, \Pi)$ should be absolutely convergent in a right half plane. We also assume that $\Pi$ is generic. (In the case that $\Pi$ is not generic, we realize it as a quotient of an induced representation of Whittaker type $\Xi$ and in what follows we use $\Xi$ in place of $\Pi$.) Then $\Pi$ has a Whittaker model $\mathcal{W}(\Pi, \psi)$ consisting of functions $W=W_{\xi}$ for $\xi \in V_{\Pi}$ that also satisfy

$$
W(u g)=\psi(u) W(g)
$$

and hence define functions on $N(k) \backslash G(\mathbb{A})$. In some sense the goal of the converse theorem is to intertwine these functions into the space of smooth automorphic forms much like the Poincaré series does for those $f \in S(N \backslash G ; \psi)$ in the classical applications.

It is easy to see that we cannot use a Poincaré series to intertwine these functions, for if we began with a cuspidal automorphic representation $\Pi$ then each vector $\varphi \in V_{\Pi}$ has a Fourier expansion of the form

$$
\varphi(g)=\sum_{\gamma \in \mathrm{N}_{n-1}(k) \backslash \mathrm{GL}_{n-1}(k)} W_{\varphi}\left(\left(\begin{array}{ll}
\gamma & \\
& 1
\end{array}\right) g\right) .
$$

Since $\varphi$ is a cusp form, we see that after averaging $W_{\varphi}$ over $\mathrm{N}_{n-1}(k) \backslash \mathrm{GL}_{n-1}(k)$ the result is already invariant under all of $\mathrm{GL}_{n}(k)$ and further averaging would thus diverge.

So, returning to our general $\Pi$, for the converse theorem we are forced to investigate what we choose to call a partial Poincaré series which, for $W \in \mathcal{W}(\Pi, \psi)$, we define by

$$
U(g)=\sum_{\gamma \in \mathrm{N}_{n-1}(k) \backslash \mathrm{GL}_{n-1}(k)} W\left(\left(\begin{array}{ll}
\gamma & \\
& 1
\end{array}\right) g\right) .
$$

This can be reformulated in terms of the mirabolic subgroup

$$
\mathrm{P}_{n}=\operatorname{Stab}_{\mathrm{GL}_{n}}((0, \ldots, 0,1))
$$

as

$$
U(g)=\sum_{p \in \mathrm{N}_{n}(k) \backslash \mathrm{P}_{n}(k)} W(p g) .
$$

Convergence is guaranteed by Propositions 12.2 and 12.3 of $[\mathbf{6}]$ (quoted as Lemma 6.1 in $[3])$.

The purpose of this note is to prove the following regularity property of these partial Poincaré series $U(g)$. We assume further that $W \in \mathcal{W}_{0}(\Pi, \psi)$, the subspace of Whittaker functions that are $K_{\infty}$-finite at the archimedean places, and that $U$ is the partial Poincaré series attached to such a $W$. Note that such Whittaker functions are dense in $\mathcal{W}(\Pi, \psi)$. 
THEOREM 1.1. Let $W \in \mathcal{W}_{0}(\Pi, \psi)$, the subspace of Whittaker functions that are $K_{\infty}$-finite at the archimedean places, and let $U(g)$ be the partial Poincaré series attached to $W$. Let $h \in \mathrm{GL}_{n-1}(\mathbb{A})$ and consider the function $U\left(\begin{array}{ll}h & \\ & 1\end{array}\right)$. Then $U\left(\begin{array}{ll}h & \\ & 1\end{array}\right)$ is a rapidly decreasing automorphic function on $\mathrm{GL}_{n-1}(\mathbb{A})$.

The careful reader will note that this statement is part of the statement of Lemma 6.2 of [3]. In the spring of 2002 we were contacted by Wee-Tek Gan, who pointed out that the proof of this statement in $[\mathbf{3}]$ in the number field case considered here was in fact "content free". We corrected the proof not long thereafter, but have never published the correction. We would like to take this opportunity to do so and at the same time place this result in the broader context of Poincaré series where it seems most natural.

Acknowledgments. We first thank Wee-Tek Gan for bringing to our attention the error in [3]. We thank S. Rallis and F. Shahidi for both moral and mathematical support in addressing this error. Most importantly we thank H. Jacquet for his help with details in this argument and for providing us with the working title for the paper ("My Remorse"). We thank Dinakar Ramakrishnan for taking interest in the new proof and encouraging us to finally publish this result in this volume dedicated to Steve Gelbart. Finally, we thank the referee for a careful checking of the details of our argument and for helping us improve the exposition is several key places.

\section{Reduction theory and the notion of rapid decrease}

As a reference for this (fairly) standard material we follow Section I.2 of [7].

Embed $\mathbb{R}_{+}^{\times}$in $\mathbb{A}^{\times}$in the standard way: to $t \in \mathbb{R}_{+}^{\times}$we associate the idele $\left(t_{v}\right)$ such that $t_{v}=t$ if $v$ is archimedean and $t_{v}=1$ for $v$ non-archimedean. If we let $\mathbb{A}^{1}=\{a \in \mathbb{A}|| a \mid=1\}$ then we can decompose $\mathbb{A}^{\times}=\mathbb{R}_{+}^{\times} \times \mathbb{A}^{1}$.

Let $\mathrm{B}_{n}$ be the standard upper-triangular Borel subgroup of $\mathrm{GL}_{n}$. Its unipotent radical is the group $\mathrm{N}_{n}$ of the introduction. Let $\mathrm{T}_{n}$ denote the diagonal torus and $\mathrm{Z}_{n}$ the center. If we write an element $a$ of $\mathrm{T}_{n}$ as a diagonal matrix $a=$ $\operatorname{diag}\left(a_{1}, \ldots, a_{n}\right)$ then the simple roots associated to $\mathrm{B}_{n}$ are $\Delta=\left\{\alpha_{1}, \ldots, \alpha_{n-1}\right\}$ where $\alpha_{i}(a)=a_{i} / a_{i+1}$.

$\mathrm{T}_{n}(\mathbb{A}) \simeq\left(\mathbb{A}^{\times}\right)^{n}$ through its matrix entries. Let $A_{n}^{+} \subset \mathrm{T}_{n}(\mathbb{A})$ be the connected subgroup $A_{n}^{+} \simeq\left(\mathbb{R}_{+}^{\times}\right)^{n} \subset\left(\mathbb{A}^{\times}\right)^{n}$. The group of rational characters of $\mathrm{T}_{n}$ is isomorphic to $\mathbb{Z}^{n}$. Viewing $\mathrm{T}_{n}$ as the diagonal torus these characters are given by

$$
\chi_{m}\left(\operatorname{diag}\left(a_{1}, \ldots, a_{n}\right)\right)=\prod a_{i}^{m_{i}}
$$

for $m=\left(m_{1}, \ldots, m_{n}\right) \in \mathbb{Z}^{n}$, which we will denote by $a \mapsto a^{m}$. Every rational character then defines a homomorphism $\chi_{m}: \mathrm{T}_{n}(\mathbb{A}) \rightarrow \mathbb{A}^{\times}$. Let

$$
\mathrm{T}_{n}^{1}(\mathbb{A})=\bigcap_{\mathbb{Z}^{n}} k e r\left|\chi_{m}\right| .
$$

We have a homomorphism $\nu: \mathrm{T}_{n}(\mathbb{A}) \rightarrow A_{n}^{+}$defined by

$$
\nu\left(\operatorname{diag}\left(a_{1}, \ldots, a_{n}\right)\right)=\operatorname{diag}\left(\left|a_{1}\right|, \ldots,\left|a_{n}\right|\right)
$$

whose kernel is precisely $\mathrm{T}_{n}^{1}(\mathbb{A})$. Thus we can decompose $\mathrm{T}_{n}(\mathbb{A})=A_{n}^{+} \mathrm{T}_{n}^{1}(\mathbb{A})$.

Let $X_{n}$ denote the group of complex characters of $\mathrm{T}_{n}(\mathbb{A})$ which are trivial on $\mathrm{T}_{n}^{1}(\mathbb{A})$. These are characters of $A_{n}^{+}$. Let $X_{n}^{\circ}$ denote the subgroup of $X_{n}$ which 
are trivial on the center $\mathrm{Z}_{n}(\mathbb{A})$ of $\mathrm{GL}_{n}(\mathbb{A})$. Finally let $Y_{n}$ denote the characters of $\mathrm{GL}_{n}(\mathbb{A})$ which are trivial on $\mathrm{GL}_{n}^{1}(\mathbb{A})=\left\{g \in \mathrm{GL}_{n}(\mathbb{A})|| \operatorname{det}(g) \mid=1\right\}$, that is , characters of the form $g \mapsto|\operatorname{det}(g)|^{s}$ for $s \in \mathbb{C}$. The real valued characters on $A_{n}^{+}$ then split as

$$
\operatorname{Re} X_{n}=\operatorname{Re} Y_{n} \oplus \operatorname{Re} X_{n}^{\circ} .
$$

The characters in $\operatorname{Re} Y_{n}$ are the real powers of the determinant, i.e., $g \mapsto|\operatorname{det}(g)|^{r}$ for $r \in \mathbb{R}$. The compliment, so the real characters of $\mathrm{T}_{n}(\mathbb{A}) / \mathrm{T}_{n}^{1}(\mathbb{A})$ which are trivial on the center $Z_{n}(\mathbb{A})$ are the products of real powers of the simple roots, i.e., are of the form

$$
a \mapsto \prod_{i=1}^{n-1}\left|\alpha_{i}(a)\right|^{r_{i}}
$$

for $\left(r_{1}, \ldots, r_{n-1}\right) \in \mathbb{R}^{n-1}$. Let us denote this character by

$$
|\alpha(a)|^{r}=\prod_{i=1}^{n-1}\left|\alpha_{i}(a)\right|^{r_{i}}
$$

with $r=\left(r_{1}, \ldots, r_{n-1}\right)$.

Fix a maximal compact subgroup $\mathrm{K}_{v} \subset \mathrm{GL}_{n}\left(k_{v}\right)$ at each place, where for the finite places we take $\mathrm{K}_{v}=\mathrm{GL}_{n}\left(\mathfrak{o}_{v}\right)$. Let $\mathrm{K}_{n}=\prod_{v} \mathrm{~K}_{v}$. We have the Iwasawa decomposition

$$
\mathrm{GL}_{n}(\mathbb{A})=\mathrm{B}_{n}(\mathbb{A}) \mathrm{K}_{n}=\mathrm{N}_{n}(\mathbb{A}) \mathrm{T}_{n}(\mathbb{A}) \mathrm{K}_{n}=\mathrm{N}_{n}(\mathbb{A}) A_{n}^{+} \mathrm{K}_{n} .
$$

Accordingly, if $g \in \mathrm{GL}_{n}(\mathbb{A})$ we can write it as $g=u a k$ with $u \in \mathrm{N}_{n}(\mathbb{A}), a \in$ $\mathrm{T}_{n}(\mathbb{A})$ and $k \in \mathrm{K}_{n}$. Write $a=a(g)$. This is not unique, but its image $\nu(a(g))$ in $\mathrm{T}_{n}(\mathbb{A}) / \mathrm{T}_{n}^{1}(\mathbb{A}) \simeq A_{n}^{+}$is. Denote this by $\nu(a(g))=|a(g)|$. If we compose this with the character $a \mapsto a^{r}$ for $r \in \mathbb{R}^{n}$ we will denote this by $|a(g)|^{r}$. If we compose this with the character $|\alpha(\cdot)|^{r^{\prime}}$ for $r^{\prime} \in \mathbb{R}^{n-1}$ defined above, we will denote this by $|\alpha(g)|^{r^{\prime}}$.

The group $\mathrm{GL}_{n}(\mathbb{A})$ carries a natural norm defined by

$$
\|g\|=\prod_{v} \sup _{i, j}\left\{\left|g_{i, j}\right|_{v},\left|\left(g^{-1}\right)_{i, j}\right|_{v}\right\}
$$

This is related to the functions $|a(g)|^{r}$ and $|\alpha(g)|^{r}$ in the following way:

(i) given $r \in \mathbb{R}^{n}$ there exist $c>0$ and $r_{0}>0$ such that

$$
|a(g)|^{r} \leq c\|g\|^{r_{0}}
$$

for all $g \in \mathrm{GL}_{n}(\mathbb{A})$;

(ii) there exists $r \in \mathbb{R}^{n-1}$ and $c>0$ such that

$$
\|g\| \leq c|\alpha(g)|^{r}
$$

for all $g \in \mathrm{GL}_{n}^{1}(\mathbb{A})$.

For any positive real number $t_{0}$ let

$$
A_{n}^{+}\left(t_{0}\right)=\left\{a \in A_{n}^{+} \mid \alpha_{i}(a)>t_{0} i=1, \ldots, n-1\right\} .
$$

By a Siegel set $\mathfrak{S}_{n} \subset \mathrm{GL}_{n}(\mathbb{A})$ is meant a set of the form $\mathfrak{S}_{n}=\omega A_{n}^{+}\left(t_{0}\right) \mathrm{K}$ where $\omega$ is a compact subset of $\mathrm{N}_{n}(\mathbb{A})$. By reduction theory for $\mathrm{GL}_{n}$ if we take $\omega$ large enough and $t_{0}$ small enough then

$$
\mathrm{GL}_{n}(k) \mathfrak{S}_{n}=\mathrm{GL}_{n}(\mathbb{A})
$$

and $\left\{\gamma \in \mathrm{GL}_{n}(k) \mid \gamma \mathfrak{S}_{n} \cap \mathfrak{S}_{n} \neq \emptyset\right\}$ is finite. We fix such a Siegel set. 
Let $Z_{n}^{+}=\mathrm{Z}_{n}(\mathbb{A}) \cap A_{n}^{+} \simeq \mathbb{R}_{+}^{\times}$. A function $\varphi: \mathfrak{S}_{n} \rightarrow \mathbb{C}$ is said to be rapidly decreasing on the Siegel set $\mathfrak{S}_{n}$ if there exists $r_{0}>0$ and for each $r \in \mathbb{R}^{n}$ there exists $c=c_{r}$ such that

$$
|\varphi(z g)| \leq c\|z\|^{r_{0}}|a(g)|^{r}
$$

for all $z \in Z_{n}^{+}$and $g \in \mathrm{GL}_{n}^{1}(\mathbb{A}) \cap \mathfrak{S}_{n}$. A function $\varphi: \mathrm{GL}_{n}(k) \backslash \mathrm{GL}_{n}(\mathbb{A}) \rightarrow \mathbb{C}$ is called rapidly decreasing if its restriction to the Siegel set $\mathfrak{S}_{n}$ is rapidly decreasing in the previous sense.

We should view $a \mapsto|a(g)|^{r}$ as an element of $\operatorname{Re} X_{n}$. For the condition of rapid decrease, when we restrict to $\mathrm{GL}_{n}^{1}(\mathbb{A}) \cap \mathfrak{S}_{n}$ we have $|\operatorname{det}(g)|=1$ and hence we need only consider characters in $\operatorname{Re} X_{n}^{\circ}$, so of the form $a \mapsto|\alpha(a)|^{r^{\prime}}$ for $r^{\prime} \in \mathbb{R}^{n-1}$. So we may rewrite the condition of rapid decay on $\mathrm{GL}_{n}(k) \backslash \mathrm{GL}_{n}(\mathbb{A})$ in the following form.

$\varphi$ is rapidly decreasing on $\mathrm{GL}_{n}(k) \backslash \mathrm{GL}_{n}(\mathbb{A})$ if there exists $r_{0}>0$ and for each $r \in \mathbb{R}^{n-1}$ there exists $c=c_{r}$ such that

$$
|\varphi(z g)| \leq c\|z\|^{r_{0}}|\alpha(g)|^{-r}
$$

for all $z \in Z_{n}^{+}$and $g \in \mathrm{GL}_{n}^{1}(\mathbb{A}) \cap \mathfrak{S}_{n}$.

Note that we have chosen to reformulate this with a negative exponent. Since the functions $|\alpha(g)|$ are positive and bounded from below on the Siegel set, it suffices to establish such estimates for $r>>0$ in the sense that all of its coordinates are large and positive. This is the form in which we shall use it in what follows.

\section{Gauges}

The standard reference for gauges and gauge estimates is the paper [6]. Gauge estimates were instrumental in proving Propositions 12.2 and 12.3 of [6] which give the convergence of our partial Poincaré series. As our proof of the rapid decay of our partial Poincaré series will grow out of these propositions, we recall here the results on gauges we will need.

A gauge on $\mathrm{GL}_{n}(\mathbb{A})$ is a non-negative function which is left invariant under the maximal unipotent subgroup $\mathrm{N}_{n}(\mathbb{A})$ and right invariant under the maximal compact $K=\mathrm{K}_{n}$, hence completely determined by its values on the maximal torus $\mathrm{T}_{n}(\mathbb{A})$, and there it is of the form

$$
\xi\left(\operatorname{diag}\left(a_{1}, \ldots, a_{n}\right)\right)=\left|\prod_{i=1}^{n-1} a_{i} / a_{i+1}\right|^{-t_{0}} \phi\left(a_{1} / a_{2}, \ldots, a_{n-1} / a_{n}\right)
$$

where $\phi\left(x_{1}, \ldots, x_{n-1}\right) \in \mathcal{S}\left(\mathbb{A}^{n-1}\right)$ is a non-negative Schwartz-Bruhat function $(\phi \geq$ $0)$ and $t_{0} \in \mathbb{R}_{+}^{\times}$. In terms of the simple roots, if $a \in \mathrm{T}_{n}(\mathbb{A})$ then

$$
\begin{aligned}
\xi(a) & =\left|\prod_{i=1}^{n-1} \alpha_{i}(a)\right|^{-t_{0}} \phi\left(\alpha_{1}(a), \ldots, \alpha_{n-1}(a)\right) \\
& =|\alpha(a)|^{-t_{0}} \phi\left(\alpha_{1}(a), \ldots, \alpha_{n-1}(a)\right)
\end{aligned}
$$

with constant power $-t_{0}$. Note that this notion makes sense over a local field, as in Section 2 of [6], but we will primarily use the global version as in Section 12 of $[6]$. $[6]$.

We will use the following two properties of gauges, proved in Lemma 12.1.5 of 
1. If $\Omega \subset \mathrm{GL}_{n}(\mathbb{A})$ is compact then there is a second gauge $\xi_{1}$ such that $\xi(g x) \leq$ $\xi_{1}(g)$ for all $g \in \mathrm{GL}_{n}(\mathbb{A})$ and $x \in \Omega$.

2. If $\Omega \subset \mathrm{GL}_{n}(\mathbb{A})$ is compact then there is a second gauge $\xi_{2}$ such that $\xi(g) \leq$ $\xi_{2}(g x)$ for all $g \in \mathrm{GL}_{n}(\mathbb{A})$ and $x \in \Omega$.

We will also have occasion to use a third property of gauges, which is Lemma 12.1.3 of $[\mathbf{6}]$.

3. Suppose $\xi$ is a gauge defined as above and that $t_{0}^{\prime}>t_{0}$ is given. Then there is a non-negative $\phi^{\prime} \in \mathcal{S}\left(\mathbb{A}^{n-1}\right)$ such that the gauge $\xi^{\prime}$ defined by $t_{0}^{\prime}$ and $\phi^{\prime}$ majorizes $\xi$.

\section{Proof of the Theorem}

Let us now turn to the proof of our theorem. In many respects it is a refinement of the arguments given in Section 12 of [6] and we will try to indicate the points of contact as the argument proceeds.

We begin with $\Pi$, an irreducible admissible generic representation of $\mathrm{GL}_{n}(\mathbb{A})$. Take $W \in \mathcal{W}_{0}(\Pi, \psi)$ and form the partial Poincaré series

$$
U(g)=\sum_{\gamma \in \mathrm{N}_{n-1}(k) \backslash \mathrm{GL}_{n-1}(k)} W\left(\left(\begin{array}{cc}
\gamma & 0 \\
0 & 1
\end{array}\right) g\right)
$$

which upon restriction to $\mathrm{GL}_{n-1}(\mathbb{A})$ gives

$$
U\left(\begin{array}{ll}
h & 0 \\
0 & 1
\end{array}\right)=\sum_{\gamma \in \mathrm{N}_{n-1}(k) \backslash \mathrm{GL}_{n-1}(k)} W\left(\begin{array}{cc}
\gamma h & 0 \\
0 & 1
\end{array}\right) .
$$

Let us use $\operatorname{diag}(h, 1)$ for $h \in \mathrm{GL}_{n-1}(\mathbb{A})$ to denote the matrix $\left(\begin{array}{ll}h & 0 \\ 0 & 1\end{array}\right) \in \mathrm{GL}_{n}(\mathbb{A})$. By applying Proposition 2.3.6 and Lemma 8.3 .3 of [6] we know that when we restrict the Whittaker function $W$ to $\mathrm{GL}_{n-1}(\mathbb{A})$ the resulting function is majorized by a gauge $\xi$ on $\mathrm{GL}_{n}(\mathbb{A})$, so that taking absolute values on each term we obtain the majorization

$$
|U(\operatorname{diag}(h, 1))| \leq \sum_{\gamma \in \mathrm{N}_{n-1}(k) \backslash \mathrm{GL}_{n-1}(k)} \xi(\operatorname{diag}(\gamma h, 1))
$$

where $\xi$ is a gauge on $\mathrm{GL}_{n}(\mathbb{A})$. Note that $\xi$ being a gauge on $\mathrm{GL}_{n}(\mathbb{A})$ will give control of all simple roots of $\mathrm{GL}_{n-1}$ as well as the center of $\mathrm{GL}_{n-1}$.

Now consider the behavior of $U(\operatorname{diag}(h, 1))$ for $h \in \mathfrak{S}_{n-1}$ a Siegel set in $\mathrm{GL}_{n-1}(\mathbb{A})$. Write $\mathfrak{S}_{n-1}=\omega_{1} A_{n-1}^{+}\left(t_{0}\right) \mathrm{K}_{n-1}$ with $\omega_{1}$ compact in $N_{n-1}(\mathbb{A})$. To investigate rapid decay on $\mathrm{GL}_{n-1}(k) \backslash \mathrm{GL}_{n-1}(\mathbb{A})$ we claim that it suffices to consider $A_{n-1}^{+}(1)$, diagonal matrices $a=\left(a_{1}, \ldots, a_{n-1}\right) \in A_{n-1}^{+}$with $a_{i} / a_{i+1} \geq 1$. we write $\omega_{1} a \mathrm{~K}_{n-1}=a\left(a^{-1} \omega_{1} a \mathrm{~K}_{n-1}\right)$ then for all $a \in A_{n-1}^{+}(1)$ we have $a^{-1} \omega_{1} a \mathrm{~K}_{n-1} \subset \Omega$ for a fixed compact subset $\Omega \subset \mathrm{GL}_{n}(\mathbb{A})$ since the action of $A_{n-1}^{+}(1)$ is non-expanding on $\omega_{1}$. Using our first property of gauges from Section 3 , this lets us write, for $h=x_{1} a k \in \mathfrak{S}_{n-1}$,

$$
\mid U\left(\operatorname{diag}(h, 1) \mid \leq \sum_{\gamma \in \mathrm{N}_{n-1}(k) \backslash \mathrm{GL}_{n-1}(k)} \xi_{1}(\operatorname{diag}(\gamma a, 1)) .\right.
$$

This proves the claim and reduces us to estimating on $A_{n-1}^{+}(1)$. 
Let $A_{n-1}^{+, 1}=A_{n-1}^{+} \cap \mathrm{GL}_{n-1}^{1}(\mathbb{A})$ and $A_{n-1}^{+, 1}(1)=A_{n-1}^{+}(1) \cap \mathrm{GL}_{n-1}^{1}(\mathbb{A})$. Then $A_{n-1}^{+}=\mathrm{Z}_{n-1}^{+} A_{n-1}^{+, 1}$. For rapid decrease, we will have different estimates on $\mathrm{Z}_{n-1}^{+}$ and $A_{n-1}^{+, 1}(1)$. Let us first take care of the center. Write our gauge $\xi_{1}$ on $\mathrm{GL}_{n}(\mathbb{A})$ as

$$
\xi_{1}\left(\operatorname{diag}\left(a_{1}, \ldots, a_{n}\right)=\prod_{i=1}^{n-1}\left|a_{i} / a_{i+1}\right|^{-t_{1}} \phi_{1}\left(a_{1} / a_{2}, \ldots, a_{n-1} / a_{n}\right) .\right.
$$

Then writing $a=z^{+} a^{1}$ for $a \in A_{n-1}^{+, 1}(1)$ we have

$$
\operatorname{diag}\left(\gamma z^{+} a^{1}, 1\right)=\operatorname{diag}\left(z^{+}, 1\right) \operatorname{diag}\left(\gamma a^{1}, 1\right) .
$$

Note that $\xi_{1}(\operatorname{diag}(z, 1))=|z|^{-t_{1}} \phi_{1}(1, \ldots, 1, z) \leq c|z|^{-t_{1}}$ and in a similar manner

$$
\xi_{1}\left(\operatorname{diag}\left(\gamma z^{+} a^{1}, 1\right)\right) \leq c|z|^{-t_{1}} \xi_{1}\left(\operatorname{diag}\left(\gamma a^{1}, 1\right)\right)
$$

Since for $z \in \mathrm{Z}^{+}$we have $|z|=\|z\|$ we see that this estimate is that which we need on the center for showing that $U(\operatorname{diag}(h, 1))$ is of rapid decrease. So now it suffices to estimate for $a \in A_{n-1}^{+, 1}(1)$.

Let us set

$$
V(a)=\sum_{\gamma \in \mathrm{N}_{n-1}(k) \backslash \mathrm{GL}_{n-1}(k)} \xi_{1}(\operatorname{diag}(\gamma a, 1))
$$

and show that this is rapidly decreasing on $A_{n-1}^{+, 1}(1)$.

As in the proof of Proposition 12.2 in [6], take a compact neighborhood of $a$ in $\mathfrak{S}_{n-1} \cap \mathrm{GL}_{n-1}^{1}(\mathbb{A})$ of the form $\omega_{a}=\omega_{1} a \omega_{3} \mathrm{~K}_{n-1}$ with $\omega_{1}$ as above and $\omega_{3}$ a compact neighborhood of 1 in $A_{n-1}^{+, 1}(1)$. As before, $a^{-1} \omega_{a} \subset \Omega^{\prime}$ with $\Omega^{\prime}$ independent of $a \in A_{n-1}^{+}(1)$. Note that the volume of $\omega_{a}$, denoted $\operatorname{Vol}\left(\omega_{a}\right)$, is a constant times a power of the modulus function $\delta(a)$ and is hence polynomial in $|\alpha(a)|$.

Take $r=\left(r_{1}, \ldots, r_{n-2}\right) \in \mathbb{R}_{+}^{n-2}$ a multi-index and recall that for $a \in A_{n-1}$ we have defined $|\alpha(a)|^{r}=\prod_{i=1}^{n-2}\left|\alpha_{i}(a)\right|^{r_{i}}$ which is $\prod\left(a_{i} / a_{i+1}\right)^{r_{i}}$ if $a \in A_{n-1}^{+}$. Consider

$$
|\alpha(a)|^{r} \operatorname{Vol}\left(\omega_{a}\right) V(a)=\sum_{\gamma \in \mathrm{N}_{n-1}(k) \backslash \mathrm{GL}_{n-1}(k)} \xi_{1}(\operatorname{diag}(\gamma a, 1))|\alpha(a)|^{r} \operatorname{Vol}\left(\omega_{a}\right) .
$$

On the right hand side we can re-write this as

$$
\begin{aligned}
\sum_{\gamma \in \mathrm{N}_{n-1}(k) \backslash \mathrm{GL}_{n-1}(k)} & \xi_{1}(\operatorname{diag}(\gamma a, 1))|\alpha(a)|^{r} \int_{\omega_{a}} d x \\
= & \int_{\omega_{a}} \sum_{\gamma \in \mathrm{N}_{n-1}(k) \backslash \mathrm{GL}_{n-1}(k)} \xi_{1}(\operatorname{diag}(\gamma a, 1))|\alpha(a)|^{r} d x .
\end{aligned}
$$

Using our second property of gauges from Section 3 and that $a^{-1} \omega_{a} \subset \Omega^{\prime}$ we may write $\xi_{1}(\operatorname{diag}(\gamma a, 1)) \leq \xi_{2}(\operatorname{diag}(\gamma x, 1))$ for all $x \in \omega_{a}$. Also, let us extend the function $|\alpha(a)|^{r}$ to a function on $\mathrm{GL}_{n-1}^{1}(\mathbb{A})$ by making it left invariant under $\mathrm{N}_{n-1}(\mathbb{A})$ and right invariant under $\mathrm{K}_{n-1}$ using the Iwasawa decomposition, i.e., to the function $|\alpha(h)|^{r}$ from Section 2. Then we may estimate the above as

$$
\begin{aligned}
\int_{\omega_{a}} \sum_{\gamma \in \mathrm{N}_{n-1}(k) \backslash \mathrm{GL}_{n-1}(k)} \xi_{1}(\operatorname{diag}(\gamma a, 1))|\alpha(a)|^{r} d x \\
\leq \int_{\omega_{a}} \sum_{\gamma \in \mathrm{N}_{n-1}(k) \backslash \mathrm{GL}_{n-1}(k)} \xi_{2}(\operatorname{diag}(\gamma x, 1))|\alpha(x)|^{r} d x .
\end{aligned}
$$


As in the proof of Proposition 12.5 of [6] (see page 229) we may replace the integral over $\omega_{a}$ with one over $\mathrm{GL}_{n-1}^{1}(k) \backslash \mathrm{GL}_{n-1}^{1}(\mathbb{A})$ at the cost of a positive constant

$$
\begin{aligned}
\int_{\omega_{a}} & \sum_{\gamma \in \mathrm{N}_{n-1}(k) \backslash \mathrm{GL}_{n-1}(k)} \xi_{2}(\operatorname{diag}(\gamma x, 1))|\alpha(x)|^{r} d x \\
& \leq c \int_{\mathrm{GL}_{n-1}^{1}(k) \backslash \mathrm{GL}_{n-1}^{1}(\mathbb{A})} \sum_{\gamma \in \mathrm{N}_{n-1}(k) \backslash \mathrm{GL}_{n-1}(k)} \xi_{2}(\operatorname{diag}(\gamma x, 1))|\alpha(x)|^{r} d x .
\end{aligned}
$$

Now replace $|\alpha(x)|^{r}$ by the Eisenstein series $E(x, r)=\sum|\alpha(\gamma x)|^{r}$ on $\mathrm{GL}_{n-1}^{1}(\mathbb{A})$. As long as $r>>0$ this converges. Assuming $r>>0$, and recalling that $\xi_{2}$ is nonnegative, we estimate the single term $|\alpha(x)|^{r}$ from above by the complete sum (of positive terms) $E(x, r)$ to obtain

$$
\begin{aligned}
\int_{\mathrm{GL}_{n-1}^{1}(k) \backslash \mathrm{GL}_{n-1}^{1}(\mathbb{A})} \sum_{\gamma \in \mathrm{N}_{n-1}(k) \backslash \mathrm{GL}_{n-1}(k)} \xi_{2}(\operatorname{diag}(\gamma x, 1))|\alpha(x)|^{r} d x \\
\leq \int_{\mathrm{GL}_{n-1}^{1}(k) \backslash \mathrm{GL}_{n-1}^{1}(\mathbb{A})} \sum_{\gamma \in \mathrm{N}_{n-1}(k) \backslash \mathrm{GL}_{n-1}(k)} \xi_{2}(\operatorname{diag}(\gamma x, 1)) E(x, r) d x .
\end{aligned}
$$

Noting that $\mathrm{GL}_{n-1}^{1}(k)=\mathrm{GL}_{n-1}(k)$, we next unfold the sum to

$$
\begin{aligned}
\int_{\mathrm{GL}_{n-1}^{1}(k) \backslash \mathrm{GL}_{n-1}^{1}(\mathbb{A})} \sum_{\gamma \in \mathrm{N}_{n-1}(k) \backslash \mathrm{GL}_{n-1}(k)} \xi_{2}(\operatorname{diag}(\gamma x, 1)) E(x, r) d x \\
=\int_{\mathrm{N}_{n-1}(k) \backslash \mathrm{GL}_{n-1}^{1}(\mathbb{A})} \xi_{2}(\operatorname{diag}(x, 1)) E(x, r) d x
\end{aligned}
$$

and perform the integration over $\mathrm{N}_{n-1}(k) \backslash \mathrm{N}_{n-1}(\mathbb{A})$ which replaces $E(x, r)$ by its constant term

$$
E_{\mathrm{N}_{n-1}}(x, r)=\sum_{w \in W_{n-1}} c(w, r)|\alpha(x)|^{w r} .
$$

Using the Iwasawa decomposition this reduces to

$$
\begin{aligned}
\int_{\mathrm{N}_{n-1}(k) \backslash \mathrm{GL}_{n-1}^{1}(\mathbb{A})} \xi_{2}(\operatorname{diag}(x, 1)) E & (x, r) d x \\
& =\sum_{w} c(w, r) \int_{A_{n-1}^{1}(\mathbb{A})} \xi_{2}(\operatorname{diag}(a, 1))|\alpha(a)|^{w r} d a .
\end{aligned}
$$

Consider now any term on the right. Let $r^{\prime}=w r$. Write

$$
\xi_{2}\left(a^{\prime}\right)=\left|\prod_{i=1}^{n-1} \alpha_{i}\left(a^{\prime}\right)\right|^{-t} \varphi\left(\alpha_{1}\left(a^{\prime}\right), \ldots, \alpha_{n-1}\left(a^{\prime}\right)\right),
$$

for $a^{\prime} \in A_{n}^{+}$using that $A_{n-1}^{+}(\mathbb{A}) \subset A_{n}^{+}(\mathbb{A})$, with $\varphi$ a Schwartz-Bruhat function and $\alpha_{i}(a)=a_{i} / a_{i+1}$. Recall

$$
|\alpha(a)|^{r^{\prime}}=\prod_{i=1}^{n-2} \alpha_{i}(a)^{r_{i}^{\prime}}
$$

Then

$$
\xi_{2}(\operatorname{diag}(a, 1))|\alpha(a)|^{r^{\prime}}=\prod_{i=1}^{n-2}\left|\alpha_{i}(a)\right|^{-t+r_{i}^{\prime}}\left|a_{n-1}\right|^{-t} \varphi\left(\alpha_{1}(a), \ldots, \alpha_{n-2}(a), a_{n-1}\right) .
$$


Now $\varphi\left(\alpha_{1}\left(a^{\prime}\right), \ldots, \alpha_{n-1}\left(a^{\prime}\right)\right)$ is rapidly decreasing as the $\alpha_{i}\left(a^{\prime}\right) \rightarrow \infty$ and is bounded as $\alpha_{i}\left(a^{\prime}\right) \rightarrow 0$. Therefore by taking $t^{\prime}=\max _{i}\left(t-r_{i}^{\prime}\right)$ with $r_{n-1}^{\prime}=0$ we see that $\xi_{2}(\operatorname{diag}(a, 1))|\alpha(a)|^{r^{\prime}}<\xi_{3}(\operatorname{diag}(a, 1))$ where

$$
\xi_{3}\left(a^{\prime}\right)=\left|\prod_{i=1}^{n-1} \alpha_{i}\left(a^{\prime}\right)\right|^{-t^{\prime}} \varphi^{\prime}\left(\alpha_{1}\left(a^{\prime}\right), \ldots, \alpha_{n-1}\left(a^{\prime}\right)\right)
$$

is another gauge on $\mathrm{GL}_{n}(\mathbb{A})$. This follows from the third property of gauges given in Section 3.

If $\xi$ is any gauge on $\mathrm{GL}_{n}(\mathbb{A})$ we know that

$$
\int_{A_{n-1}(\mathbb{A})} \xi(\operatorname{diag}(a, 1))|\operatorname{det}(a)|^{s} d a
$$

is convergent for $\operatorname{Re}(s)>>0$. This follows from factoring the integral as a product

$$
\prod_{v} \int_{A_{n-1}\left(k_{v}\right)} \xi_{v}\left(\operatorname{diag}\left(a_{v}, 1\right)\right)\left|\operatorname{det}\left(a_{v}\right)\right|_{v}^{s} d a_{v}
$$

and using the expression for the determinant in terms of roots. Next decompose $A_{n-1}(\mathbb{A})=A_{+} \times A_{n-1}^{1}(\mathbb{A})$ where $A_{+} \subset A_{n-1}(\mathbb{R})$ with $A_{+} \simeq \mathbb{R}_{+}^{\times}$. Then taking $s=\sigma$ to be real and sufficiently large to guarantee absolute convergence, we have

$$
\begin{aligned}
& \int_{A_{n-1}(\mathbb{A})} \xi(\operatorname{diag}(a, 1))|\operatorname{det}(a)|^{\sigma} d a \\
&=\int_{A_{+}}\left(\int_{A_{n-1}^{1}(\mathbb{A})} \xi\left(\operatorname{diag}\left(a^{+} a^{1}, 1\right)\right) d a^{1}\right)\left(a^{+}\right)^{\sigma} d a^{+} .
\end{aligned}
$$

So by Fubini the inner integral is finite for every $a^{+}$and in particular

$$
\int_{A_{n-1}^{1}(\mathbb{A})} \xi\left(\operatorname{diag}\left(a^{1}, 1\right)\right) d a^{1}<\infty .
$$

Tracing our way back, this last step implies that each

$$
\int_{A_{n-1}^{+, 1}(\mathbb{A})} \xi_{2}(\operatorname{diag}(a, 1))|\alpha(a)|^{w r} d a
$$

is finite, and hence

$$
\int_{\mathrm{GL}_{n-1}^{1}(k) \backslash \mathrm{GL}_{n-1}^{1}(\mathbb{A})} \sum_{\gamma \in \mathrm{N}_{n-1}(k) \backslash \mathrm{GL}_{n-1}(k)} \xi_{2}(\operatorname{diag}(\gamma x, 1)) E(x, r) d x
$$

is finite. Thus

$$
\int_{\mathrm{GL}_{n-1}^{1}(k) \backslash \mathrm{GL}_{n-1}^{1}(\mathbb{A})} \sum_{\gamma \in \mathrm{N}_{n-1}(k) \backslash \mathrm{GL}_{n-1}(k)} \xi_{2}(\operatorname{diag}(\gamma x, 1))|\alpha(x)|^{r} d x
$$

is finite and

$$
\sum_{\gamma \in \mathrm{N}_{n-1}(k) \backslash \mathrm{GL}_{n-1}(k)} \int_{\omega_{a}} \xi_{1}(\operatorname{diag}(\gamma a, 1))|\alpha(a)|^{r} d x
$$

is finite. This finally implies that for each $r>>0$

$$
|\alpha(a)|^{r} \operatorname{Vol}\left(\omega_{a}\right) V(a)
$$


is finite and so

$$
V(a)=\sum_{\gamma \in \mathrm{N}_{n-1}(k) \backslash \mathrm{GL}_{n-1}(k)} \xi_{1}(\operatorname{diag}(\gamma a, 1)) \leq c|\alpha(a)|^{-r} \operatorname{Vol}\left(\omega_{a}\right)^{-1} \leq c^{\prime}|\alpha(a)|^{-r^{\prime}}
$$

for every $r^{\prime}>>0$.

As a consequence we obtain, for every $r^{\prime}>>0$,

$$
|U(\operatorname{diag}(z h, 1))| \leq c\|z\|^{-t_{1}}|\alpha(h)|^{-r^{\prime}}
$$

for $h \in \mathrm{GL}_{n-1}^{1}(\mathbb{A}) \cap \mathfrak{S}$. But this is rapid decrease of the partial Poincaré series $U(\operatorname{diag}(h, 1))$ on $\mathrm{GL}_{n-1}(k) \backslash \mathrm{GL}_{n-1}(\mathbb{A})$ as desired.

\section{The Converse Theorem}

To get an intertwining into the space of smooth automorphic forms, as we did in the case of Poincaré series, we need more arithmetic input. This is the content of the converse theorems for $\mathrm{GL}_{n}$. For completeness, let us paraphrase the basic converse theorem from $[\mathbf{3}]$ in terms of our partial Poincaré series.

TheOREM 5.1. Let $\Pi \simeq \otimes^{\prime} \Pi_{v}$ be an irreducible admissible (generic) representation of $\mathrm{GL}_{n}(\mathbb{A})$ whose central character is automorphic and whose formally defined L-function

$$
L(s, \Pi)=\prod_{v} L\left(s, \Pi_{v}\right)
$$

is absolutely convergent in some right half plane. Suppose in addition that for every $m$ with $1 \leq m \leq n-1$ and for every cuspidal automorphic representation $\tau$ of $\mathrm{GL}_{m}(\mathbb{A})$ the twisted $L$-functions $L(s, \Pi \times \tau)$ are nice, i.e.,

(1) both $L(s, \Pi \times \tau)$ and $L(1-s, \tilde{\Pi} \times \tilde{\tau})$ extend to entire functions of $s$;

(2) these extensions are bounded in vertical strips of finite width;

(3) they satisfy the basic functional equation

$$
L(s, \Pi \times \tau)=\varepsilon(s, \Pi \times \tau) L(1-s, \tilde{\Pi} \times \tilde{\tau}) .
$$

Then the partial Poincaré series

$$
U(g)=\sum_{\gamma \in \mathrm{N}_{n-1}(k) \backslash \mathrm{GL}_{n-1}(k)} W\left(\left(\begin{array}{ll}
\gamma & 0 \\
0 & 1
\end{array}\right) g\right)
$$

for $W \in \mathcal{W}_{0}(\Pi, \psi)$ defines a smooth cusp form on $\mathrm{GL}_{n}(\mathbb{A})$, and so, in particular, is left invariant under $\mathrm{GL}_{n}(k)$.

For stronger versions of this result see Theorem 2 and Theorem 3 of $[\mathbf{3}]$ and Theorem 1 and Theorem 2 of [4], which allow more restricted twisting sets, both in terms of rank of the groups and in terms of ramification of the twisting representations. However, the proofs of all of these results begin with this same basic partial Poincaré series.

\section{References}

[1] J.W. Cogdell, J-S. Li, I.I. Piatetski-Shapiro, and P. Sarnak, Poincaré series for $\mathrm{SO}(n, 1)$. Acta Math. 167 (1991), 229-285.

[2] J.W. Cogdell and I.I. Piatetski-Shapiro, The Arithmetic and Spectral Analysis of Poincaré Series. Perspectives in Mathematics, Vol.13. Academic Press, Boston, 1990.

[3] J.W. Cogdell and I.I. Piatetski-Shapiro, Converse theorems for $G L_{n}$. Publ. Math. IHES 79 (1994), 157-214. 
[4] J.W. Cogdell and I.I. Piatetski-Shapiro, Converse theorems for $G L_{n}$, II. J. Reine Angew. Math. 507 (1999), 165-188.

[5] J.W. Cogdell, I.I. Piatetski-Shapiro, and P. Sarnak, Estimates on the critical line for Hilbert modular L-functions and applications, in preparation.

[6] H. Jacquet, I.I. Piatetski-Shapiro, and J. Shalika, Automorphic forms on GL(3), I \& II. Ann. Math. 109 (1979), I: 169-212; II: 213-258.

[7] C. Moeglin and J-L. Waldspurger, Spectral Decomposition and Eisenstein Series. Cambridge Tracts in Mathematics 113. Cambridge University Press, Cambridge, 1995.

[8] A. Selberg, On the estimation of Fourier coefficients of modular forms. Proc. Symp. Pure Math. VIII (1965), 1-15.

Department of Mathematics, Ohio State University, Columbus, OH 43210

E-mail address: cogdell@math.ohio-state.edu

Department of Mathematics, Yale University, New Haven CT 06520

E-mail address: ilya@math.yale.edu 